\title{
Acolhimento com classificação de risco a pacientes suspeitos de covid-19 em unidade de pronto atendimento: relato de experiência
}

\section{Accommodation with risk classification to suspected patients of covid-19 in an erms: experience report}

DOI: $10.46919 / \operatorname{archv2n3-031~}$

Recebimento dos originais: 01/01/2021

Aceitação para publicação: 31/03/2021

\section{Romario Henrique da Silva \\ Graduando em Enfermagem.}

Instituição: Faculdade CESMAC do Sertão (FCS)

Endereço: R. Bráulio Montenegro, 285/286, Vila Maria, Palmeira dos Índios, AL, Brasil

E-mail: henrique_romario@outlook.com

Emanuelly Kesley De Freitas Lima

Graduanda em Enfermagem.

Instituição: Faculdade CESMAC do Sertão (FCS)

Endereço: R. Bráulio Montenegro, 285/286, Vila Maria, Palmeira dos Índios, AL, Brasil

E-mail: manufr11999@hotmail.com

\section{Marcela Porangaba Lopes}

Graduanda em Enfermagem.

Instituição: Faculdade CESMAC do Sertão (FCS)

Endereço: R. Bráulio Montenegro, 285/286, Vila Maria, Palmeira dos Índios, AL, Brasil

E-mail: porangabamarcela@gmail.com

Natanna Weslane Ferreira dos Santos

Enfermeira. Especialista em Enfermagem do Trabalho pelo Centro de Ensino Superior Arcanjo Mikael de Arapiraca, Alagoas, Brasil.

Especialista em Urgência, Emergência e UTI pela Faculdades Integradas de Patos, Paraíba, Brasil.

Especialista em Educação em Saúde pela Universidade Estadual de Ciências da Saúde de Alagoas, Alagoas, Brasil.

Endereço: R. Castelo Branco, 2096, Eucalipto, Palmeira dos Índios, AL, Brasil

E-mail: natxyy@hotmail.com

\section{Fernanda Pinto da Silva Farias}

Enfermeira. Especialista em Urgência, Emergência e UTI pela Faculdades Integradas de Patos, Paraíba, Brasil.

Pós-graduanda em Oncologia e Hematologia pela Faculdade de Ensino Regional Alternativa, Alagoas, Brasil.

Endereço: R. Irmãos Barbosa, 155, Eucalipto, Palmeira dos Índios, AL, Brasil

E-mail: nandapintosilva@ hotmail.com

\section{Kátia Danielle de Cerqueira Leite Gama}

Enfermeira. Especialista em Urgência, Emergência e UTI pela Faculdade Tiradentes, Brasil.

Especialista em Educação em Saúde pela Universidade Estadual de Ciências da Saúde de Alagoas, Alagoas, Brasil. 
Especialista em Ginecologia e Obstetrícia pela Faculdade Venda Nova do Imigrante, Espírito Santo, Brasil.

Endereço: Rua Major Cícero de Góes Monteiro, 102, Centro, Palmeira dos Índios, AL, Brasil

E-mail: katiagamaleite@gmail.com

\section{Rudja Maria Leite de Abreu}

Enfermeira. Mestre em Ensino na Saúde pela Universidade Federal de Alagoas, Alagoas, Brasil. Professora do curso de Enfermagem da Faculdade CESMAC do Sertão, Alagoas, Brasil. Instituição: Faculdade CESMAC do Sertão (FCS)

Endereço: R. Bráulio Montenegro, 285/286, Vila Maria, Palmeira dos Índios, AL, Brasil E-mail: rudja.abreu@cesmac.edu.br

\section{Jaqueline Maria da Silva}

Zootecnia. Pós-doutorado em Química e Biotecnologia pelo Instituto de Química e Biotecnologia da Universidade Federal de Alagoas, Alagoas, Brasil.

Professora do curso de Enfermagem da Faculdade CESMAC do Sertão, Alagoas, Brasil. Líder do grupo de pesquisa Biotecnologia e Tecnologias Aplicadas à Saúde - Biotectas

Instituição: Faculdade CESMAC do Sertão (FCS)

Endereço: R. Bráulio Montenegro, 285/286, Vila Maria, Palmeira dos Índios, AL, Brasil E-mail: jaqueline.silva@cesmac.edu.br

\section{RESUMO}

Objetivo: Descrever um relato de experiência, acerca do acolhimento com classificação de risco em uma Unidade de Pronto Atendimento a pacientes suspeitos de COVID-19. Metodologia: Trata-se de um relato de experiência, de caráter descritivo e abordagem qualitativa sobre o atendimento realizado a usuários suspeitos de COVID-19. Resultados e Discussão: O acolhimento com classificação de risco é uma ferramenta essencial que possibilita triar adequadamente os pacientes que necessitam de atendimento de acordo com suas limitações, e observando o grau de complexidade de cada caso, pode-se notar que a educação em saúde, neste processo tem se tornado mais um aliado no atual cenário da pandemia, visto que o acolhimento com classificação de risco permite ao profissional direcionar cuidados e até mesmo explicalos. Com isso foi-se necessário adotar o fluxograma que facilita o atendimento. Considerações Finais: Esta vivência permitiu concluir que o enfermeiro durante o acolhimento tem adotado fluxogramas que facilitam, direcionam e agilizam o atendimento a pacientes suspeitos ou confirmados da infecção. Do mesmo modo que outras ferramentas como a educação em saúde tem se mostrado eficaz no momento da classificação.

Palavras-Chave: Emergência, Infecções por Coronavírus, Acolhimento, Enfermagem, Educação em Saúde.

\section{INTRODUÇÃO}

Os serviços de urgência e emergência são elementos fundamentais da assistência à saúde (MENDES et al., 2018). Entre os componentes da Rede de Atenção as Urgências, estão as Unidades de Pronto Atendimento (UPA 24h) e o conjunto de serviços de urgência 24 horas não hospitalares que conforme a portaria $\mathrm{N}^{\circ} 1.600 / 2011$ :

[...] devem prestar atendimento resolutivo e qualificado aos pacientes acometidos por quadros agudos ou agudizados de natureza clínica e prestar primeiro atendimento aos casos de natureza cirúrgica ou de trauma, estabilizando os pacientes e realizando a investigação diagnóstica inicial, 
definindo, em todos os casos, a necessidade ou não, de encaminhamento a serviços hospitalares de maior complexidade (PORTARIA, 2011, p. 5).

A COVID-19 se propagou ligeiramente no mundo inteiro, sua expansão trouxe impactos econômicos, sociais e para a saúde. Essa doença progrediu em todos os continentes, por isso atribuiu a necessidade de contenção e isolamento de pessoas para diminuir o aumento do número de pessoas infectadas (SANTOS, 2021).

Os usuários têm utilizado as UPAs não somente para casos de urgência ou de emergência, mas também como uma forma de suprir a lacuna presente entre a resolutividade e assistência na atenção primária e ambulatorial brasileira (RONCALLI et al., 2017). Já esperado o comportamento por parte da população observado a cima, ao se tratar do cenário atual de pandemia pelo novo Coronavírus as pessoas diante do aparecimento de qualquer queixa ou sintoma desloca-se até esses serviços em busca de orientações e atendimento.

Afim de organizar o fluxo de usuários e melhorar a qualidade da assistência, o Ministério da Saúde (MS) implantou o Acolhimento com Classificação de Risco (ACCR), proporcionando maior agilidade nos atendimentos com atenção às necessidades do paciente e promovendo humanização e redução de riscos (BRASIL, 2009).

O ACCR tem objetivos como: acolher; classificar o risco do agravo; direcionar a clientela ao atendimento médico de acordo com a gravidade do caso; informar o paciente que não corre risco imediato, assim como a seus familiares, sobre o tempo provável de espera; diminuir a insatisfação dos usuários e profissionais, tendo em vista que agiliza o serviço e, principalmente, estimular a pactuação e a construção de redes internas e externas de atendimento, fazendo os encaminhamentos necessários (DIAS; SANTOS; SILVA, 2018).

De acordo com o Conselho Federal de Enfermagem em sua resolução No 423/ 2012:

No âmbito da equipe de Enfermagem, a classificação de risco e priorização da assistência em Serviços de Urgência é privativa do Enfermeiro, observadas as disposições legais da profissão. [...] o Enfermeiro deverá estar dotado dos conhecimentos, competências e habilidades que garantam rigor técnico-científico ao procedimento (COFEN, 2012, p.2).

Realizada durante a primeira etapa do processo de enfermagem, a Classificação de Risco é de responsabilidade do enfermeiro, que com base em um protocolo já preestabelecido classifica o usuário em um sistema de cores (FREITAS et al., 2017).

As unidades de pronto atendimento ofertam diversos serviços, no entanto o cenário pandêmico da COVID-19, permitiu acrescentar mais uma patologia a este serviço. A COVID-19 trata-se de uma doença causada pelo coronavírus denominado SARS-CoV-2 (ZHU et al., 2020) ocasionando síndrome respiratória, foi inicialmente detectada em 2019 na cidade de Wuhan, capital da província da China Central. Tem 
atingido diferentes níveis de complexidade, sendo os casos mais graves acometidos de uma insuficiência respiratória aguda que requer cuidados hospitalares intensivos - incluindo o uso de ventilação mecânica (Centers for Disease Control and Prevention [CDC], 2020b).

A atual pandemia pelo Coronavírus tem superlotado os serviços de emergência, tornando necessário que os enfermeiros atuantes na classificação de risco estejam preparados para atender essa nova demanda, fornecendo as orientações e encaminhamentos necessários para que a assistência seja efetiva. O enfermeiro deve estar em constante aprendizado, analisando as melhores maneiras de realização do cuidado e educação em saúde, enfatizando a importância das medidas de proteção individual e coletiva, evitando a disseminação do vírus, tanto em ambiente hospitalar como comunitário (THOMAS et al., 2020).

Assim nota-se que a educação em saúde, deve ser considerada como estratégia importante mediante tal cenário. Neste contexto, a educação em saúde compõe um conjunto de práticas e saberes orientados para a prevenção de doenças e promoção da saúde (ALVES, 2005). Trata-se de uma forma de educar que deve ser repensada e valorizada como uma tecnologia de trabalho, possibilitando diversas formas de levar ao usuário informações relevantes, tomando como princípios os preceitos do SUS e uma aprendizagem significativa, promovendo mudança na vida dos envolvidos, assim como na realidade do modelo de atenção à saúde vigente (PINAFO; NUNES; GONZALES, 2012).

Á vista disso vale ressaltar a importância do ACCR em meio ao cenário mundial pandêmico. Objetivando assim, descrever um relato de experiência, acerca do acolhimento com classificação de risco em uma Unidade de Pronto Atendimento - UPA a pacientes suspeitos de COVID-19.

\section{METODOLOGIA}

Trata-se de um relato de experiência, de caráter descritivo e abordagem qualitativa sobre o atendimento realizado a usuários suspeitos de COVID-19, vivenciado pelos discentes do curso de enfermagem da Faculdade Cesmac do Sertão e enfermeiras assistenciais atuantes, que resultou em uma atividade de educação em saúde utilizando o ACCR na Unidade de Pronto-atendimento (UPA) direcionada a pacientes suspeitos de COVID-19, localizada no bairro Vila Maria, em um município do Sertão alagoano.

A atividade ocorreu do dia 27 de maio de 2020 a 29 de julho, no setor de atendimento da UPA, a classificação de risco utilizada ACCR foi a estabelecida pelo ministério da saúde adaptada do protocolo de Manchester, de acordo com a gravidade dos casos clínicos.

A ação se estabeleceu em 1 manhã por semana nos meses, por meio de uma conversa informativa, rápida e sucinta ressaltando a importância de prevenção e ação recomendada em caso de acometimento pelo vírus, seguindo todas as medidas de biossegurança de acordo com a nota técnica da ANVISA (GVIMS/GGTES/ANVISA No 04/2020). 
A vivência constitui-se de: Acolhimento do usuário com classificação de risco, atendimento de enfermagem, momento de orientação e educação em saúde. Este relato compreendeu os meses de maio, junho e julho do ano de 2020, período em que ocorreram mudanças e adaptações no referido serviço, de maneira a tornar possível realizar atendimentos direcionados aos pacientes suspeitos e/ou acometidos pela COVID-19.

\section{RESULTADOS E DISCUSSÃO}

O ambiente desta vivência refere-se a UPA com atendimento $24 \mathrm{~h}$, estando identificada como porte II, possuindo em seu quadro, médicos clínicos, enfermeiros, assistentes sociais, técnicos em enfermagem, farmacêuticos, auxiliar em farmácia e técnicos em raio x. Devido ao contexto de pandemia, a unidade passou por adequações físicas, ocorrendo ampliação da unidade com inclusão de novos leitos, profissionais e equipamentos que formam a área COVID-19, para onde são encaminhados casos confirmados e suspeitos identificados durante a triagem ou casos confirmados mediante comprovação de exame que identifique a presença do vírus no paciente.

A UPA funciona 24 horas, durante os sete dias da semana. Fazem parte da Rede de Urgência e Emergência e tem por objetivo prestar atendimento de saúde de complexidade intermediária, atuando em conjunto com a Atenção Básica (AB), atenção hospitalar, atenção domiciliar e o Serviço de Atendimento Móvel de Urgência (SAMU) (BORDIGNON et al., 2020).

Diante do cenário de pandemia, o sistema de classificação de risco precisou passar por adequações, afim de garantir a segurança do profissional e paciente suspeito ou acometido por COVID-19, para tal foram seguidos fluxogramas de atendimento estabelecidos pelo Ministério da Saúde.

A UPA referida adotou um fluxograma que permite triar pacientes com maior agilidade, e realizar o acolhimento de risco adequado para cada caso. Distribuído da seguinte maneira:

Pacientes que se enquadram na síndrome gripal, estejam com SPO2 maior ou igual a 95\%, são direcionados para um local adequado, seguindo as normas do ministério da saúde, separados dos demais pacientes, afim de evitar aglomerações. Onde ficam aguardando atendimento médico.

Pacientes com SPO2 menor que 95\%, mais síndrome gripal ou síndrome respiratória aguda grave, são direcionados automaticamente para a ala Covid, na qual é dividida em partes: suspeitos (síndrome gripal estável) não confirmados que necessitam de oxigênio, pacientes com Síndrome Gripal mais graves, e pacientes confirmados, todas as alas são separadas segundo normas do ministério da saúde.

O protocolo de manejo do Covid-19 (Ministério da Saúde, 2020) preconiza que o reconhecimento precoce de pacientes suspeitos, incluindo aqueles com doenças graves, possibilita o início oportuno de medidas adequadas, tratamentos de suporte otimizados, encaminhamento e a admissão segura e rápida para 
leito de enfermaria hospitalar ou unidade de terapia intensiva designada para este fim, de acordo com os protocolos institucionais ou nacionais.

$\mathrm{O}$ acolhimento se deu pela realização da anamnese, exame físico e verificação dos sinais vitais, sendo este processo efetuado de forma direcionada para identificação do quadro do paciente. A anamnese teve como foco: Sinais e sintomas com ênfase no sistema respiratório, história de viagem recente e contato com caso confirmado, sendo a seguir verificado os sinais vitais e oximetria de pulso afim de observar os níveis de saturação de oxigênio no sangue. Com base na avaliação inicial o paciente enquadrando-se como suspeito ou confirmado é classificado conforme a prioridade de atendimento mediante preenchimento de ficha disponível no sistema informatizado da unidade, sendo conduzido a uma sala de isolamento para paciente suspeitos ou para setor de confirmados.

Segundo o Ministério da Saúde (2020) as complicações mais comuns são síndrome respiratória aguda grave (SRAG)definida por presença de dispneia ou os seguintes sinais de gravidade: Saturação de $\mathrm{SpO} 2$ < que 95\% em ar ambiente; sinais de desconforto respiratório ou aumento da frequência respiratória avaliada de acordo com a idade; piora nas condições clínicas de doença de base; hipotensão em relação à pressão arterial habitual do paciente; indivíduo de qualquer idade com quadro de insuficiência respiratória aguda durante o período sazonal. A apresentação de alteração nos níveis de saturação e frequência respiratória é considerado sinal de gravidade, portanto é encaminhado de imediato para atendimento.

Durante o atendimento destaca-se realização da orientação quanto aos sinais e sintomas da COVID19, medidas de prevenção como: utilização de máscara, distanciamento social, higienização das mãos, etiqueta respiratória, como também, observação de sinais ou sintomas de agravamento e esclarecimento de dúvidas. Tal ação contribuiu para elucidação de questionamentos por parte do paciente e acompanhantes, notando-se diminuição dos sinais de medo e angustia.

Frente a situação, se fez necessário capacitação dos profissionais atuantes no serviço com o propósito de garantir atendimento integral, humanizado e eficaz, para tal ocorreu: treinamentos, reuniões multiprofissionais e disponibilização dos fluxogramas na unidade. Cabendo ao profissional ter uma visão holística e integral de maneira a prestar uma assistência de qualidade e humanizada. Como pode ser corroborado por Soncini, Molina e Soares (2021) que expõe a pandemia da Covid-19 trouxe muitas situações novas para os profissionais de saúde, que passaram a ter de lidar comas mudanças de protocolos de atendimentos quase diários, com uma nova carga de roupas e equipamentos de proteção, com a privação do toque e da proximidade durante os atendimentos e com o medo de se contaminar. Essas e outras mudanças tiveram que ser absorvidas rapidamente pelos profissionais para serem colocadas em prática.

Assim, observa-se que a devida aplicação do fluxograma de atendimento, é importante para o serviço por facilitar e organizar o mesmo garantindo atendimento rápido e eficaz. Com isso a educação em 
saúde de forma permanente e/ou continuada é uma ferramenta valiosa para que o serviço tenha êxito na assistência de qualidade e implantação de protocolos.

\section{CONSIDERAÇÕES FINAIS}

Em face do exposto percebe-se que o acolhimento com classificação de risco nesse momento de pandemia permite identificar precocemente sinais, e sintomas que se enquadrem clinicamente na COVID19, possibilitando direcionar o cuidado adequado para cada caso.

Nota-se que algumas medidas preventivas têm se tornado de grande importância, evidenciadas ainda no momento da classificação, pois pode-se observar que a educação em saúde vem se tornando uma ferramenta essencial, na prevenção, tratamento e diagnóstico.

Vale ressaltar que a educação em saúde não é uma ferramenta nova praticada na área, mas que nesse momento de pandemia observa-se que vem tomando grandes proporções, as informações constantes sobre o atual cenário vêm surtindo efeito positivo na população, embora o numero de casos ainda não esteja estabilizado. Porém, constata-se que alguns cuidados estão se tornando rotina como uso de mascaras, e higienização constante das mãos com álcool.

Assim, infere-se que as vivências relatadas refletem as contribuições positivas e importantes para o atual cenário mundial. A experiência relatada mostra que, embora em um período crítico para o sistema de saúde devido à pandemia pelo COVID-19, é possível/ necessário a realização de adaptações e mudanças conforme a necessidade que o momento apresenta. Os profissionais receberam capacitação para o desempenho adequado do protocolo em questão, permitindo melhor fluxo de atendimento, prezando pela integralidade da assistência e segurança profissional-paciente. Evidenciando ainda, o protagonismo da Enfermagem, a capacidade que esses profissionais possuem de adequar os cenários de atenção à saúde em todos os níveis, bem como, a efetividade em seu papel de educador em saúde. 


\section{REFERÊNCIAS}

ALVES, Vânia Sampaio. Um modelo de educação em saúde para o Programa Saúde da Família: pela integralidade da atenção e reorientação do modelo assistencial. Interface - Comunic., Saúde, Educ., v.9, n.16, p.39-52, set.2004/fev. 2005. https://doi.org/10.1590/S1414-32832005000100004.

Agencia Estatal Boletín Oficial del Estado. (2020b). Real Decreto 487/2020, de 10 de abril, por el que se prorroga el estado de alarma declarado por el Real Decreto 463/2020, de 14 de marzo, por el que se declara el estado de alarma para la gestión de la situación de crisis sanitaria ocasionada por el COVID-19. Boletín Oficial del Estado Madrid: Autor. Recuperado el http://www.boe.es/eli/es/rd/2020/04/10/487

BORDIGNON, Juliana Silveira et al. Vivências e autonomia de enfermeiras de uma Unidade de Pronto Atendimento em tempo de pandemia. Enferm. foco (Brasília), p. 205-210, 2020.

BRASIL. Ministério da Saúde. Protocolo de manejo clínico da Covid-19 na Atenção Especializada. Secretaria de Atenção Especializada à Saúde, Departamento de Atenção Hospitalar, Domiciliar e de Urgência. 1. ed. rev. Brasília: Ministério da Saúde, 2020.

BRASIL. Ministério da Saúde. Secretaria de Atenção à Saúde. Política Nacional de Humanização da Atenção e Gestão do SUS. Brasília: Ministério da Saúde, 2009. 56 p.: il. color. - (Série B. Textos Básicos de Saúde).

BRASIL. Portaria $n^{\circ} 1.600$, de 7 de Julho de 2011. Reformula a Política Nacional de Atenção às Urgências e institui a Rede de Atenção às Urgências no Sistema Único de Saúde (SUS). Diário Oficial da União, 8 jul. 2011., Seção 1.

BRASIL. ANVISA (Agência Nacional de Vigilância Sanitária). NOTA TÉCNICA GVIMS/GGTES/ANVISA N ${ }^{\circ}$ 07/2020, Orientações para a prevenção da transmissão de Covid-19 dentro dos serviços de saúde. Brasília. 08 maio. 2020.

CONSELHO FEDERAL DE ENFERMAGEM - COFEN. Resolução 423/2012. [online]. 2015. Disponível em: http://www.cofen.gov.br/resoluo-cofen-n-4232012_8956.html. Acesso em: 20, maio 2021.

DIAS, S. R. S; SANTOS, L. L; SILVA, I. A. Classificação de risco no serviço de urgência e emergência: revisão integrativa da literatura. Rev Enferm UFPI. 2018 Jan-Mar;7(1):57-62.

FARO, A.; BAHIANO, M. A.; NAKANO, T. C.; REIS, C.; SILVA, B. F. P.; VITTI, L. S. COVID-19 e saúde mental: a emergência do cuidado. Estudos de Psicologia (Campinas), 37, e200074. 2020. https://doi.org/10.1590/1982-0275202037e200074

FREITAS, R. J. M; PEREIRA, M. F. A; LIMA, C. H. P; MELO, J. N; OLIVEIRA, K. K. D. A violência contra os profissionais da enfermagem no setor de acolhimento com classificação de risco. Rev Gaúcha Enferm. 2017;38(3): e 62119.

MENDES, T. S. M; SILVEIRA, L. M; SILVA, L. P; STABILE, A. M. Associação entre o acolhimento com classificação de risco, desfecho clínico e o Escore Mews. REME Rev Min Enferm. 2018; 22: e-1077.

PINAFO, E; NUNES, E. F. P. A; GONZÁLES, A. D. A educação em saúde na relação usuário-trabalhador no cotidiano de equipes de saúde da família. Ciência \& Saúde Coletiva, 7(7):1825-1832, 2012. 
RONCALLI, A. A; OLIVEIRA, D. N; SILVA, I. C. M; BRITO, R. F; VIEGAS, S. M. F. Protocolo de Manchester e população usuária na classificação de risco: visão do enfermeiro. Rev. baiana enferm. 31(2): 16949, 2017.

SANTOS, Juliana Soares Laudelino. Tempos Pandêmicos: A Covid-19 E Seus Impactos Para A População Socialmente Vulnerável. Archives of Health, v. 2, n. 3, p. 258-262, 2021. DOI: https://doi.org/10.46919/archv2n3-005

SONCINI, M. D.; MOLINA, L. R.; SOARES, L. Processo de trabalho e produção do cuidado na Atenção Primária à Saúde durante a pandemia de COVID-19 em Florianópolis-SC: experiências vivenciadas por uma farmacêutica residente. Archives of Health, v. 2, n. 3, p. 401-421, 2021. DOI: https://doi.org/10.46919/archv2n3-017

THOMAS, L. S; PIETROWSKI, K; KINALSKI, S. S; BITTENCOURT, V. L. L; SANGOI, K. C. M. Atuação do enfermeiro emergencista na pandemia de covid-19: Revisão narrativa da literatura. Braz. J. Hea. Rev., Curitiba, v. 3, n. 6, p. 15959-15977 nov./dez. 2020.

YIN, R. K. Estudo de caso: planejamento e métodos. 4ª ed. Porto Alegre (RS): Bookman; 2010.

ZHU, N; ZHANG, D; WANG, W; LI, X; YANG. B; SONG, J, et al. A novel coronavirus from patients with pneumonia in China, 2019. N Engl J Med [Internet]. 2020. DOI: http://doi.org/10.1056/NEJMoa2001017 